\title{
Amino-terminated diamond surfaces: Photoelectron emission and photocatalytic properties
}

\author{
Di Zhu, Jason A. Bandy, Shuo Li, and Robert J. Hamers* \\ Department of Chemistry, University of Wisconsin-Madison, 1101 University Avenue, \\ Madison, WI
}

"Corresponding author: rjhamers@wisc.edu

\begin{abstract}
We report a new approach to making stable negative electron-affinity diamond surfaces by terminating diamond with amino groups (also known as amine groups, $-\mathrm{NH}_{2}$ ). Previous studies have shown that negative electron affinity can be induced by terminating diamond surfaces with hydrogen, creating a surface dipole favorable toward electron emission. Here, we demonstrate that covalent tethering of positive charges in the form of protonated amino groups, $-\mathrm{NH}_{3}{ }^{+}$, also leads to negative electron affinity (NEA) and facile electron emission into vacuum and into water. Amino-terminated diamond was prepared using a very mild plasma discharge. Valence-band photoemission studies of the amino-terminated diamond samples show a characteristic "NEA" peak, demonstrating that the amino-terminated surface has NEA. Diamond's ability to emit electrons into water was evaluated using photochemical conversion of $\mathrm{N}_{2}$ to $\mathrm{NH}_{3}$. Time-resolved surface photovoltage studies were used to characterize charge separation at the diamond interface, and Mott-Schottky measurements were performed to characterize band-bending at the diamond-water interface. XPS studies show that the amino-terminated surfaces provide increased chemical resistance to oxidation compared with H-terminated diamond when illuminated with ultraviolet light.
\end{abstract}

Keywords: diamond; photocatalysis; amine; amino, electron emission; solvated electrons 


\section{Introduction}

Diamond is a wide-bandgap semiconductor with a bandgap of $5.5 \mathrm{eV}$. Because of its chemical stability and ability to be doped into a conductive state, diamond has been used as a high-stability substrate and electrode [1,2]. One unusual property of diamond is that its conduction band lies higher in energy than the vacuum level. This alignment of energy levels is referred to as Negative Electron Affinity (NEA) [3-6]. NEA materials can have unusually high electron emission yields because while in conventional materials photoelectron emission is a surface-dominated process, in NEA materials electrons that are optically excited to the conduction band in the bulk material can diffuse to the surface and be emitted with virtually no barrier [7]. While NEA is often considered a property of the bulk energy levels, true NEA and the accompanying facile electron emission from diamond require both downward band-bending near the surface and a surface termination whose associated surface dipole favors electron emission $[8,9]$. NEA of diamond has typically been achieved by terminating the surface with hydrogen; the resulting $\mathrm{C}-\mathrm{H}$ surface dipole has a small positive charge on the $\mathrm{H}$ atom, thereby yielding a surface dipole oriented in a direction that favors electron emission [6-8].

While electron emission into vacuum has been studied for many years, electron emission into water is much less studied. Yet, it is very important because the emission into water leads to the formation of solvated electrons, long regarded as the chemist's perfect reducing agent [10], and because solvated electrons in water can induce many exceedingly difficult reactions of importance in chemistry and biology [7,11-13]. However, until recently there has not been a convenient way of producing solvated electrons in water. Most previous studies formed solvated electrons by using high-energy arcs, energetic laser pulses, or high-energy radioactive particles [10]. In recent work, we showed that diamond's NEA and high chemical stability allows it to be used as a solid-state source of solvated electrons in water, and that these electrons can induce novel reactions such as the reduction of $\mathrm{N}_{2}$ to $\mathrm{NH}_{3}[14,15]$, the reduction of $\mathrm{H}^{+}$to neutral atomic hydrogen $(\mathrm{H} \bullet)[16]$, and the one-electron reduction of $\mathrm{CO}_{2}$ to its radical anion [17]. However, we also found that the electron-emissive properties of $\mathrm{H}$-terminated diamond decreased over a duration of several hours due to oxidation of the surface [14]. 
While $\mathrm{H}$-terminated diamond has negative electron affinity because of the $\mathrm{C}-\mathrm{H}$ surface dipole, alternative strategies for forming NEA surfaces in water also exist. Chemical functional groups containing one $\mathrm{N}$ atom with three single bonds to any combination of $\mathrm{H}$ and $\mathrm{C}$ atoms are referred to as amino groups. Primary amino groups (one bond to the surface, $\mathrm{C}-\mathrm{NH}_{2}$ ) and secondary amino groups (two bonds to the surface, $\mathrm{C}_{2}-\mathrm{NH}$ ) are of particular interest because at circumneutral $\mathrm{pH}$ values in water they can be protonated to form to form positively charged quaternary cations at the diamond surface such as $\mathrm{C}-\mathrm{NH}_{3}{ }^{+}$(one bond to the surface) and $\mathrm{C}_{2}-\mathrm{NH}_{2}{ }^{+}$ (two bonds to the surface) analogous to the aqueous ammonium ion $\left(\mathrm{NH}_{4}{ }^{+}\right)$. In principle, the presence of protonated amino groups on the diamond surface could enhance electron emission because static, positive surface charge should produce downward band-bending within the adjacent diamond region and a strong electrostatic driving force for electron emission.

Here, we report investigations of the electron emission properties of amino-terminated diamond surfaces. We demonstrate that very low-power ammonia plasmas can form diamond surfaces terminated with primary amino groups. Our results show that diamond surfaces terminated with amino groups exhibit negative electron affinity in vacuum and that the emission is enhanced by intentional protonation of the surface. The amino-terminated surfaces are capable of emitting electrons into water and initiating reduction of $\mathrm{N}_{2}$ to $\mathrm{NH}_{3}$ in a manner similar to that of H-terminated diamond. XPS studies indicate that amino-terminated surfaces are more resistant to oxidation than the corresponding H-terminated surfaces.

\section{Materials and Methods}

\subsection{Preparation of H-terminated diamond.}

Electrochemical grade boron-doped diamond (Element Six) was H-terminated by exposure to a hydrogen plasma [18] in a $2.45 \mathrm{GHz}$ microwave enhanced plasma CVD chamber. In the process, the sample was kept in $\mathrm{H}_{2}$ at a pressure of 45 torr with a microwave power of $600 \mathrm{~W}$ for $15 \mathrm{~min}$. The microwave power was reduced to zero over a time period of several minutes, and the H-terminated diamond was left in the $\mathrm{H}_{2}$ atmosphere for another 15 min to cool near room temperature. 


\subsection{Preparation of amino-terminated diamond.}

To form amino-terminated diamond, we exposed $\mathrm{H}$-terminated diamond samples to an ammonia plasma in a customized radio-frequency (RF) plasma chamber consisting of a $6 \mathrm{~cm}$ diameter quartz tube surrounded by a 3-turn RF coil, connected to an impedance-matching network and a 13.56 MHz RF source. Compared to previous studies using ammonia plasmas that have typically used RF power of $300-500$ watts $[19,20]$ or microwave excitation [21], we use much lower power to minimize fragmentation. To optimize formation of surface amino groups, we measured the concentration of surface nitrogen using XPS, and the surface density of primary amino groups $\left(\mathrm{C}-\mathrm{NH}_{2}\right)$ using the SDTB method described below. We monitored the plasma composition via optical emission spectroscopy (OES), using an Ocean Optics spectrometer to characterize optical emission from the $\left(\mathrm{A}^{3} \Pi \rightarrow \mathrm{X}^{3} \Sigma^{-}\right)$emission band of $\mathrm{NH}$ at $337 \mathrm{~nm}$ and the $\left(\mathrm{C}^{3} \Pi_{\mathrm{u}} \rightarrow \mathrm{B}{ }^{3} \Pi_{\mathrm{g}}\right)$ emission band of $\mathrm{N}_{2}$ at $316 \mathrm{~nm}, 337$ and $358 \mathrm{~nm}$ [22]. At low RF powers and high pressures, the emission intensity from $\mathrm{NH}$ increased related to that of $\mathrm{N}_{2}$ due to reduced fragmentation and increased collisional quenching. We found the best conditions for amino termination to be the highest pressure and lowest RF power (in our system, 1.6 torr pressure and 25 Watts RF power) that would maintain a stable plasma. Amino termination was complete within 10 minutes under these conditions. In some cases diamond samples were intentionally protonated by immersing the samples in concentrated hydrochloric acid (36 weight $\%$ ) for one minute and then drying completely in a gentle flow of pure nitrogen gas. XPS measurements of the surface chlorine yielded a N/Cl atom ratio of 2.8 , with a surface $\mathrm{Cl}$ coverage of 6.2 atoms $/ \mathrm{nm}^{2}$.

\subsection{Measurement of primary amino groups.}

We measured the density of primary amino $\left(\mathrm{R}-\mathrm{NH}_{2}\right)$ groups via derivatization with sulfo-succinimidyl-4-O-(4,4'-dimethoxytrityl)-butyrate (sulfo-SDTB); we refer to this method of analysis as the "SDTB method" $[23,24]$. Samples of interest were immersed in a $0.1 \mathrm{mM}$ solution of sulfo-SDTB in $50 \mathrm{mM} \mathrm{NaHCO}$ at $\mathrm{pH} 8.5$ for $30 \mathrm{~min}$. Under these conditions, the $\mathrm{N}$-hydroxysulfosuccinimidyl ester reacts with the surface amino groups to form an amide bond linking the dimethoxytrityl group to the surface. After rinsing with deionized water, a 20 
$\mu \mathrm{L}$ droplet of 4.1 M trifluoroacetic acid was dripped onto the sample to release the light-absorbing dimethoxytrityl (DMT) groups into solution. The concentration of released DMT groups was determined by measuring the optical absorbance at $498 \mathrm{~nm}$ in a semimicro cuvette, and comparing with primary amine standards of known concentration.

\subsection{X-ray and Ultraviolet photoelectron spectroscopy.}

X-ray photoelectron spectroscopy (XPS) data were obtained using a custom-built ultrahigh vacuum ( $\mathrm{P}<6 \times 10^{-10}$ torr) Physical Electronics system equipped with an aluminum $\mathrm{K}_{\alpha}$ source, a quartz-crystal X-ray monochromator, and a 16-channel detector array. XPS measurements were performed using a pass energy of $58.7 \mathrm{eV}($ resolution $=0.88 \mathrm{eV})$. Quantitative measurement of nitrogen and chlorine were determined by curve-fitting and measuring the areas of the appropriate peaks. Since the $\mathrm{N}$ and $\mathrm{Cl}$ layers are monolayer or sub-monolayer coverage, there is negligible scattering within the adsorbed surface layer. In this case the number density can be obtained using the equation [25]:

$$
\mathrm{N}_{\mathrm{X}}=-\rho_{\mathrm{C}} \cdot \lambda_{\mathrm{C}} \cdot\left(\frac{\mathrm{S}_{\mathrm{C}}}{\mathrm{S}_{\mathrm{X}}}\right)\left(\frac{\mathrm{A}_{\mathrm{X}}}{\mathrm{A}_{\mathrm{C}}}\right) \cos (\theta) .
$$

Where $\rho_{\mathrm{C}}$ is the number density of carbon atoms in diamond $\left(=1.76 \times 10^{23}\right.$ atoms $\left.\mathrm{cm}^{-3}\right), \lambda_{\mathrm{C}}$ is the inelastic mean free path of $\sim 1200 \mathrm{eV}$ electrons in diamond (2.0 $\mathrm{nm}[26]), \mathrm{S}_{\mathrm{C}}$ and $\mathrm{S}_{\mathrm{X}}$ are the instrument-specific sensitivity factors for $C$ and other elements of interest $\left(S_{C}=0.296\right.$, $\mathrm{S}_{\mathrm{N}}=0.477$, and $\mathrm{S}_{\mathrm{O}}=0.711$ [27]), $\mathrm{A}_{\mathrm{X}}$ and $\mathrm{A}_{\mathrm{C}}$ are the measured integrated peak areas corresponding to element " $\mathrm{X}$ " and of $\mathrm{C}$ in the bulk diamond, respectively, and $\theta$ is the angle of emission of the detected electrons measured with respect to the surface normal (here, $45^{\circ}$ ).

Ultraviolet photoemission spectroscopy (UPS) measurements were performed with the same apparatus, using excitation from a $\mathrm{He}(\mathrm{I})$ resonance lamp (photon energy $=21.2 \mathrm{eV}$ ) and an analyzer pass energy of $2.59 \mathrm{eV}$ (resolution $=0.039 \mathrm{eV}$ ). During UPS measurements, we applied a bias of up to $-9.0 \mathrm{~V}$ to the sample to compensate for differences in work function between sample and analyzer and to ensure that all emitted electrons would be detected by the analyzer [28]; we then subtracted the applied bias from the measured electron kinetic energies. 


\subsection{Transient Surface Photovoltage (SPV) measurements of interfacial charge}

separation. Transient surface photovoltage measurements [29] used a pulsed laser (NT340, EKSPLA, Inc., Vilnius, Lithuania) to produce wavelength-tunable excitation pulses (ns pulse width, 20 pulses/sec, typically $\sim 0.1 \mathrm{~mJ} /$ pulse). These pulses were directed on the face of the diamond sample. A second capacitively coupled transparent electrode (Pt mesh) was placed $\sim 75 \mu \mathrm{m}$ away. This "sense" electrode was connected directly to the input of a $2 \mathrm{GHz}$ amplifier (50 ohms in put impedance, Gain=10, Model TA2000B-1, FAST ComTec GmbH, Oberhaching/München, Germany) and the output voltage was measured on a $4 \mathrm{GHz}$ digital oscilloscope (Agilent Model DSO9404A).

\section{Results}

XPS measurements of the plasma-treated surface yielded a surface N coverage of $17.3 \pm 2.0$ atoms $/ \mathrm{nm}^{2}$. Nitrogen can form a number of different surface species that satisfy its common coordination number of 3 for neutral species. These include primary $\left(\mathrm{C}-\mathrm{NH}_{2}\right)$ and secondary amino groups $\left(\mathrm{C}_{2} \mathrm{NH}\right)$ with one and two bonds to the surface (where " $\mathrm{C}$ " represents a surface carbon atom), cyano groups, $(-\mathrm{C} \equiv \mathrm{N})$, and imino groups $(-\mathrm{C}=\mathrm{NH}$ and $-\mathrm{C}=\mathrm{N}-\mathrm{C})$ [21]. Of these different species, primary amino groups $\left(\mathrm{C}-\mathrm{NH}_{2}\right)$ are among the most stable and most readily protonated to form positively charged surface groups $\left(-\mathrm{NH}_{3}{ }^{+}\right)$. To directly confirm the presence of primary amino groups we also used the SDTB method described above. Because the SDTB method requires covalently functionalizing the surface amino groups with a bulky molecule having a radius of $\sim 0.65 \mathrm{~nm}$ (based on molecular modeling of the length of a single arm of the trityl group within the SDTB molecule ), it saturates at molecular densities higher than $\sim 0.6$ groups $/ \mathrm{nm}^{2}$, corresponding to the steric limit (often referred to as the "jamming limit") [30] for the SDTB molecule on the surface [23]. Our SDTB measurements yielded a value corresponding to $0.6 \mathrm{NH}_{2}$ groups $/ \mathrm{nm}^{2}\left(6 \times 10^{13} \mathrm{~cm}^{-2}\right)$, confirming that the surface has a high density of primary amino groups at least this high. While the SDTB measurement can only provide a lower limit on the number of primary amino groups, the resulting lower limit is important because it establishes that if these groups can be protonated, the resulting surface sheet charge density of $>6 \times 10^{14} \mathrm{~cm}^{-2}$ is expected to be higher than the values of $\sim 10^{13}-10^{14} \mathrm{~cm}^{-2}$ required to control the sub-surface band-bending 


\subsection{Electron emission into vacuum:}

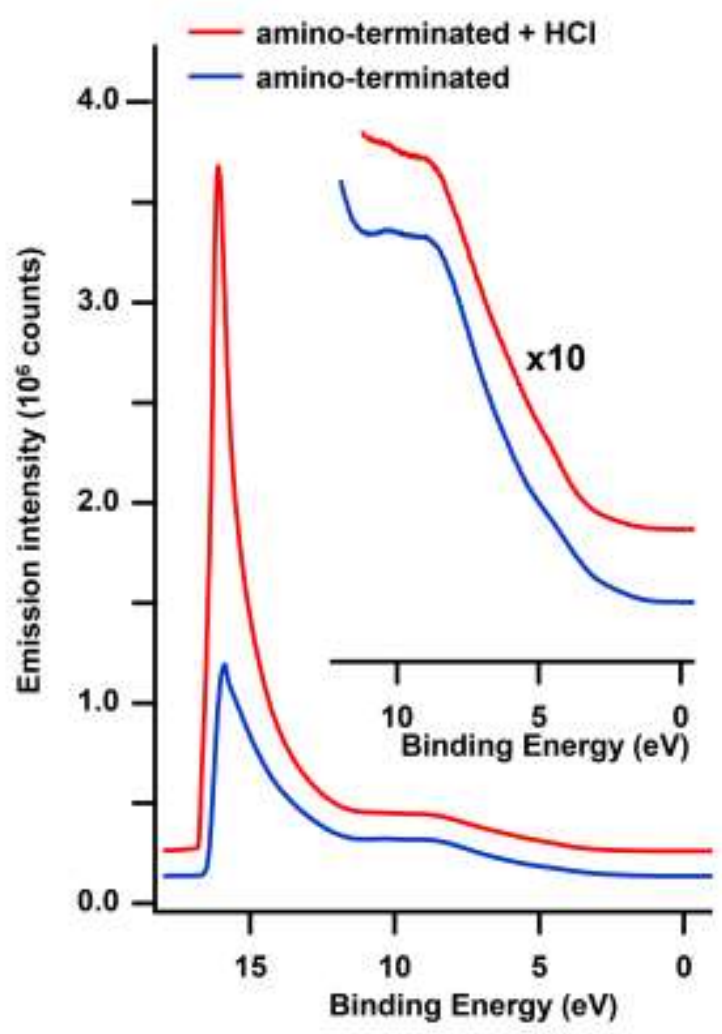

Fig. 1. Ultraviolet photoemission spectra from amino-terminated diamond, before and after brief immersion in $\mathrm{HCl}$. The inset shows the same data magnified 10x.
We first characterized the electron emission from $\mathrm{NH}_{2}$-terminated samples into vacuum. Because amino groups can be present in both neutral $-\mathrm{NH}_{2}$ and in protonated form $\left(-\mathrm{NH}_{3}{ }^{+}\right.$, with a corresponding anion $)$, we investigated the effect of exposure to $\mathrm{HCl}$, which forcibly protonates the amino groups to form $\left(-\mathrm{NH}_{3}{ }^{+} \mathrm{Cl}^{-}\right)$ion pairs. In water these ion pairs dissociate. Prior studies of protonated, amino-terminated surfaces have indicated that the surfaces consist of $-\mathrm{NH}_{3}{ }^{+}$ groups, with the $\mathrm{Cl}^{-}$counter-ions and/or $\mathrm{OH}^{-}$ions (from the water itself) separated from these positive charges by a layer of water molecules [32]. Consequently, even though the surface positive charge is balanced by negatively charged anions,

electrons at the diamond surface are in closer proximity to the positively charged $\mathrm{NH}_{3}{ }^{+}$groups, and the charge-compensating negative ions are located a larger distance way in the aqueous medium.

Figure 1 shows UPS spectra from an $\mathrm{NH}_{2}$-terminated diamond samples before and after forced protonation, which was achieved by immersing the sample in concentrated hydrochloric acid (36 weight\%) for one minute and then drying in pure nitrogen gas. Both samples show a characteristic strong increase in emission intensity near $17 \mathrm{eV}$, plummeting to nearly zero at slightly higher binding energies. This feature is particularly apparent on the $\mathrm{HCl}$-treated sample. This intense peak at high binding energy is characteristic of negative electron affinity materials and is caused by electrons excited in the bulk that relax to the 
conduction-band edge, leading to emission of electron in a narrow energy window at the energy of the conduction-band $[3,33]$. Both samples also show a weak peak near $8.5 \mathrm{eV}$ that is associated with the diamond bulk valence band [33]. Even when expanded 10x (Fig. 1, inset) the two samples have nearly identical intensities for the diamond bulk states in the 5-10 $\mathrm{eV}$ range, but the $\mathrm{HCl}$-treated sample has much higher emission near $17 \mathrm{eV}$ binding energy

\subsection{Surface photovoltage characterization of interfacial charge separation}

a)

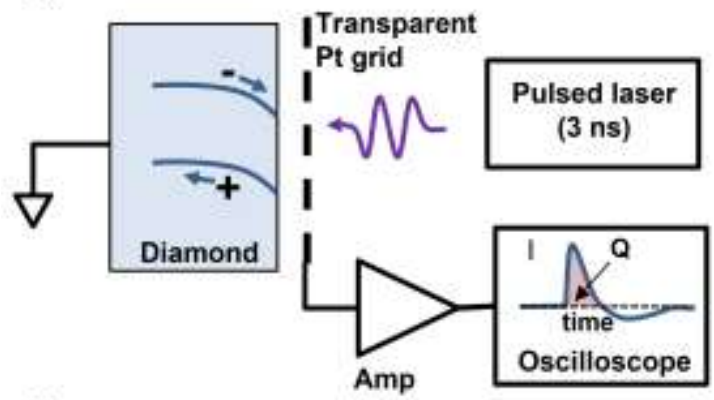

b)

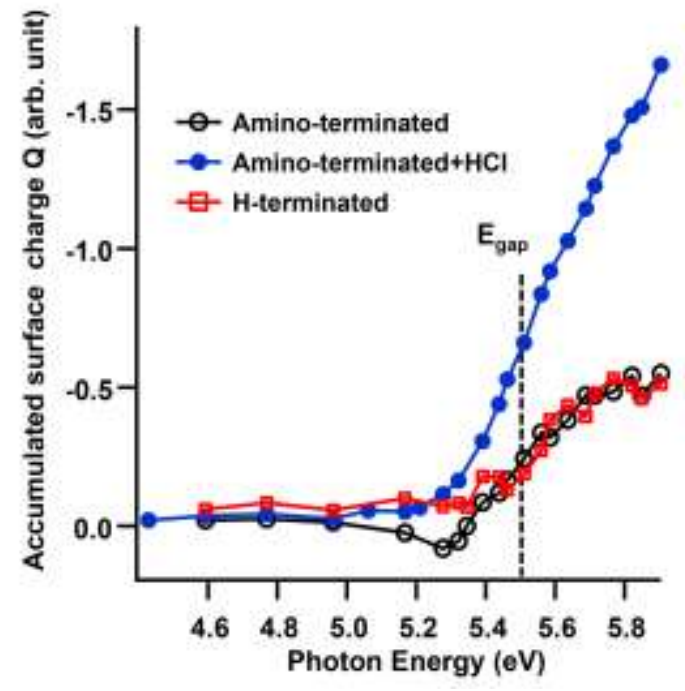

Fig 2. Surface photovoltage measurements. (a) schematic of apparatus; (b) Plots of photo-initiated charge separation (Q) as a function of UV photon energy from amino-terminated B-doped diamond before and after acidification, measured at UV pulse energy of $0.1 \mathrm{~mJ}$. The dashed line shows the $5.5 \mathrm{eV}$ bandgap energy of diamond.
In principle, greater band-bending in the sub-surface space charge region can improve charge separation and the ability to emit electrons. In order to verify that amino-termination impacts the ability to separate charge near diamond surfaces, we used transient surface photovoltage (SPV) measurements on functionalized diamond samples. Surface photovoltage measurements characterize the amount and polarity of excess charges that accumulate at the outermost surface of the sample as a result of optical excitation [34 ].

Fig. 2a depicts the experimental arrangement as implement in our experiments [29]. The sample was illuminated with a tunable, pulsed laser. The absorption of above-bandgap light

produces mobile electrons and holes near the surface. Mobile charges can also be produced by at smaller photon energies by excitations to/from surface states and bulk states within the bandgap (typically arising from defects or impurities). In both cases, charges that are mobile 
are separated by the electric field in the diamond space-charge layer, leading to a transient surface photovoltage; this voltage generates an associated transient displacement current $\mathrm{I}(\mathrm{t})$ in a second, capacitively coupled "sense" electrode (Pt mesh) placed $\sim 75 \mu \mathrm{m}$ away. The sign of the displacement current reveals whether electrons or holes accumulate near the surface. For above-bandgap excitation (which creates both mobile electrons and mobile holes) the sign of the displacement current $\mathrm{I}(\mathrm{t})$ is controlled by whether the bands bend upward or downward at the surface. Integrating the $I(t)$ curve yields the amount of charge separation $Q(t)$ as a function of time after the laser pulse. Due to the capacitive coupling, $I(t)$ has an initial sign and amplitude associated with the photo-excited charge separation, and then reverses sign as recombination processes occur and charge flows in the other direction. Consequently, $Q(t)$ has a maximum corresponding to the maximum amount of charge separation. We refer to this maximum as $\mathrm{Q}_{\max }$. Figure $2 \mathrm{~b}$ summarizes measurements of $\mathrm{Q}_{\max }$ as a function of the incident photon energy for H-terminated diamond and amino-terminated diamond. We also show results for a sample that was intentionally exposed to $\mathrm{HCl}$ in an effort to ensure that the surface amino groups were in their protonated form $\left(-\mathrm{NH}_{3}{ }^{+}\right)$.

For H-terminated diamond, no SPV response was observed for photon energies $<5.4 \mathrm{eV}$. At higher photon energies, the SPV signal demonstrates accumulation of negative charge (i.e., electrons) at the surface, which is revealed in Fig. $2 b$ as an upward-trending response. For photon energies $>5.4 \mathrm{eV}$ the amino-terminated sample shows a response very similar to that of the $\mathrm{H}$-terminated sample, but for photon energies in the 5.1-5.4 eV range the amino-terminated diamond shows a small accumulation of positive charge at the surface. Finally, the amino-terminated sample that was exposed to $\mathrm{HCl}$ shows a threshold at $5.4 \mathrm{eV}$ and significantly larger accumulation of surface negative charge surface compared with the other samples. We attribute this enhancement of charge separation to the fact that the positive surface charge induced by protonation of the amino groups increases the downward band-bending (as also evidenced from the Mott-Schottky plots) that drives excess electrons to the surface. At lower photon energies of $5.2 \mathrm{eV}$ to $5.3 \mathrm{eV}$, the amino-terminated sample shows a small wavelength region where the sign inverts, indicating that the surface accumulates positive charge. This most likely arises from excitation of electrons from normally-filled 
surface states into the conduction band [34].

\subsection{Influence of amino- termination on}

\section{diamond-water interface:}

To explore the influence of amino

termination on the surface electronic

structure, we used electrochemical

impedance spectroscopy and

Mott-Schottky measurements $[35,36]$ to

characterize the interfacial capacitance as

a function of potential in $0.1 \mathrm{M} \mathrm{KCl}$

solution. First, we identified the

open-circuit potential (OCP), which

establishes the Fermi level of the sample

with respect to the $\mathrm{Ag} / \mathrm{AgCl}$ reference

electrode. We measured an OCP of 1.01

V for amino-terminated diamond

compared with $0.24 \mathrm{~V}$ for H-terminated

diamond. The significantly more positive

value for the amino-terminated diamond

shows that in water its Fermi energy is

higher in energy (closer to the vacuum

level) than that of H-terminated diamond.

Impedance spectroscopy measurements

were used to investigate the interfacial

electrical properties. Fig. 3a shows the

impedance of H-terminated and

$-\mathrm{NH}_{2}$-terminated diamond samples as a
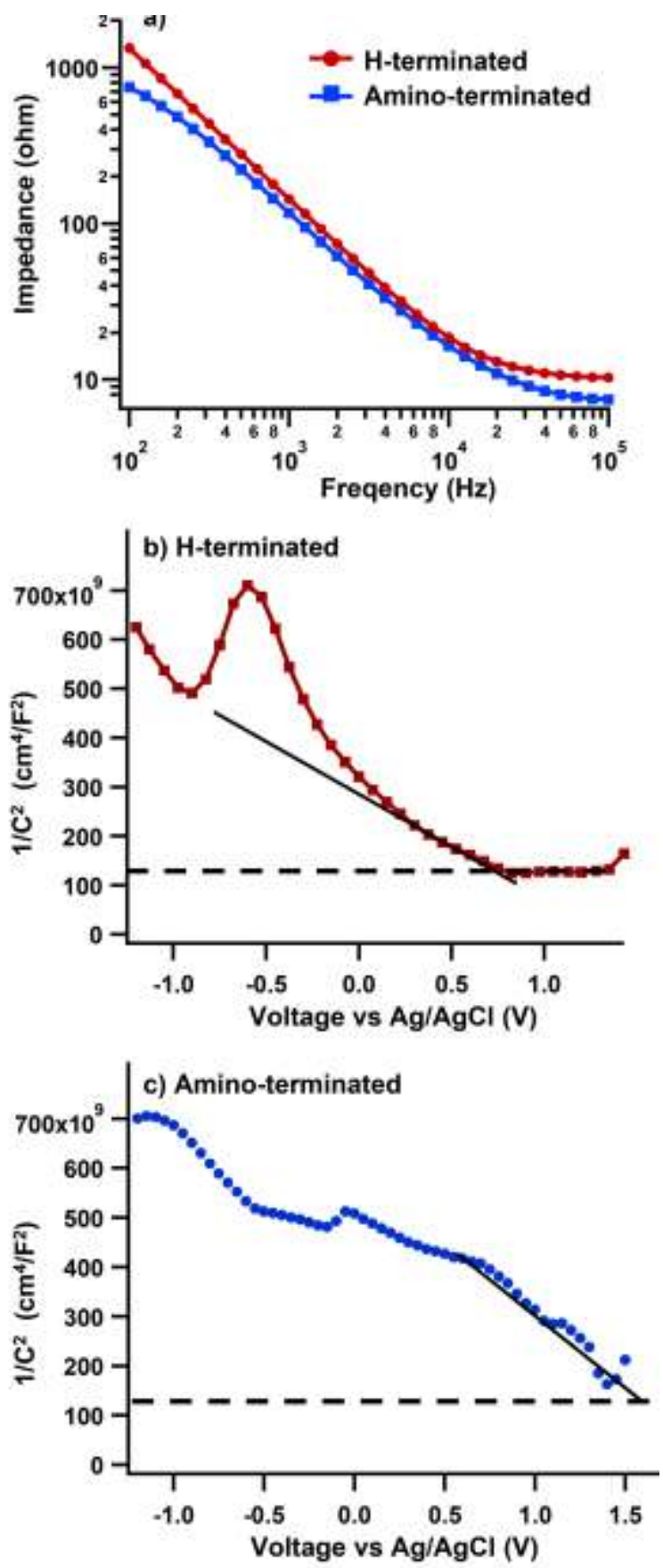

Figure 3 Electrochemical Impedance Spectroscopy of

H-terminated and amino-terminated diamond in $0.1 \mathrm{M}$

$\mathrm{KCl}$ solution a) Impedance vs. frequency, showing

1/f-dependence in the region near $2 \mathrm{kHz}$. (b,c)

Mott-Schottky measurements of the (b)

hydrogen-terminated and (c) amino-terminated

surfaces, both measured at $2 \mathrm{kHz}$.

function of frequency. At frequencies between $800 \mathrm{~Hz}$ and $10 \mathrm{kHz}$ the impedance $\mathrm{Z}$ for both 
samples decreases linearly with increasing frequency $f$; this functional dependence indicates that in this frequency range the impedance is dominated by the space-charge capacitance of the diamond sample. We modeled the complex impedance as a simplified Randles cell [37], in which the space-charge region is modeled as a parallel resistor and capacitor, and the solution resistance is simple resistor. Figure 3 also shows the conventional representation of Mott-Schottky data as a plot of $1 / \mathrm{C}^{2}$ vs. potential for H-terminated diamond (Fig. 3b) and for amino-terminated diamond (Fig. 3c). For the H-terminated sample, in Fig. 3b, the graph shows $1 / \mathrm{C}^{2}$ decreasing as the potential is made more positive, a peak near $-0.5 \mathrm{~V}$, and with a plateau at the highest potentials. This general appearance is similar to that reported previously [38] and is characteristic of a p-type semiconductor with surface defects. The large feature near $-0.5 \mathrm{~V}$ can be attributed to charging of a surface state, which has been reported previously [38]. Assuming that interfacial resistances are small (as verified from the impedance spectra), the total interfacial capacitance can be represented analytically as [39] $\frac{1}{\mathrm{C}^{2}}=\frac{1}{\mathrm{C}_{\mathrm{DL}}^{2}}+\frac{2}{\aleph_{0} \mathrm{eN}_{\mathrm{A}} \mathrm{A}^{2}}\left(\mathrm{~V}-\mathrm{V}_{\mathrm{FB}}-\frac{\mathrm{kT}}{\mathrm{e}}\right)$

where $\mathrm{N}_{\mathrm{A}}$ is the concentration of electrically active acceptors (dopants), $\mathrm{V}$ is the applied potential, $\mathrm{V}_{\mathrm{FB}}$ is the flat band potential and $\mathrm{C}_{\mathrm{DL}}$ is the double-layer capacitance of the aqueous phase, which is nearly independent of potential at the high salt concentrations used here [40]. The primary consequence of the double-layer capacitance is that when $\mathrm{V}$ is large, $\frac{1}{\mathrm{C}^{2}}$ approaches the limiting value of $\frac{1}{\mathrm{C}_{\mathrm{DL}}^{2}}$. In our case, $\frac{1}{\mathrm{C}_{\mathrm{DL}}^{2}}=1.26 \times 10^{11} \mathrm{~cm}^{4} \mathrm{~F}^{-2}$ or $\mathrm{C}_{\mathrm{DL}}=2.8$ $\mu \mathrm{F} / \mathrm{cm}^{2}$, represented by the horizontal dashed line in Fig. 2b. For the H-terminated sample, the intercept of the solid line and dashed line yields the flat band potential approximately $0.7 \mathrm{~V}$ vs. $\mathrm{Ag} / \mathrm{AgCl}$. This is similar to the value of $0.4 \mathrm{~V}$ reported previously for H-term B-doped diamond grown on Si [41]. Fig. 3c shows a Mott-Schottky experiment on amino-terminated diamond conducted under identical experimental conditions. The plot is clearly shifted toward higher (more positive) potentials. As the potential is increased beyond $+1.5 \mathrm{~V}$ there is a clear onset of electrochemical reactions evidenced visually by the appearance of bubbles from water oxidation, and the complex impedance plots change dramatically. However, if it assumed that the double-layer capacitance is the same as that for the H-terminated sample, 
then a flat-band potential of approximately $+1.5 \mathrm{~V}(\mathrm{vs} . \mathrm{Ag} / \mathrm{AgCl}$ ) is obtained. The

Mott-Schottky measurements indicate the both H-terminated and amino-terminated diamond samples are p-type with downward band-bending; the amino-terminated surface has a significantly more positive flat-band potential, demonstrating a more pronounced downward band-bending.

\subsection{Electron emission into water: Photocatalytic reduction of $\mathbf{N}_{2}$ to $\mathrm{NH}_{3}$.}

To determine whether electron emission would persist in water, we characterized the ability of amino-terminated diamond samples to induce photochemical reduction of $\mathrm{N}_{2}$ to $\mathrm{NH}_{3}$ when illuminated with ultraviolet light. These experiments were performed in a dual-compartment cell while applying a small $(-0.5 \mathrm{~V})$ bias to the diamond with respect to the open-circuit potential. Figure 4 shows the $\mathrm{NH}_{3}$ yield from a sample of $\mathrm{H}$-terminated diamond, a sample of $\mathrm{NH}_{2}$-terminated diamond, and a sample of $\mathrm{NH}_{2}$-terminated diamond that was briefly immersed in $0.1 \mathrm{M} \mathrm{HCl}$. The $\mathrm{NH}_{2}$-terminated diamond gives a slightly higher $\mathrm{NH}_{3}$ yield than

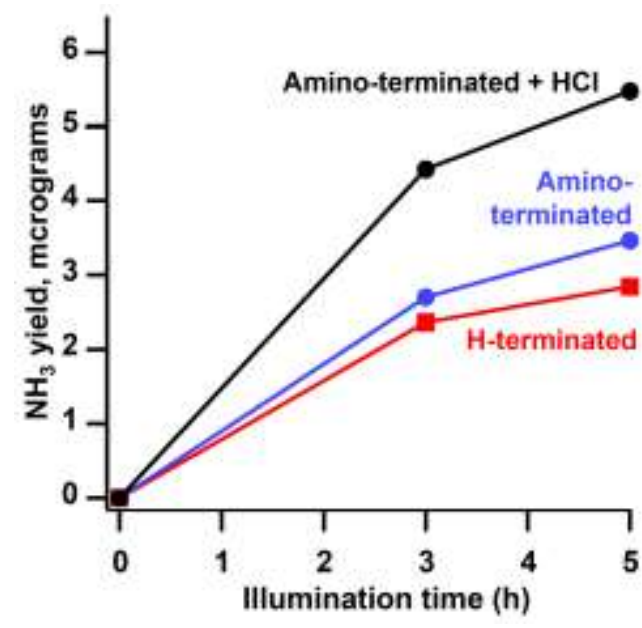

Fig. 4. Ammonia yield results for H-terminated and for amino-terminated diamond before and after exposure to $\mathrm{HCl}$. the H-terminated sample does, while the

$\mathrm{NH}_{2}$-terminated sample that was also acidified gives a significantly higher yield of $\mathrm{NH}_{3}$. These data indicate that electron emission into water follows the same general trend as that observed in vacuum.

We note that the sample has approximately 17.3 $\mathrm{N}$ atoms $/ \mathrm{nm}^{2}$ and an exposed sample area of $\sim 0.3 \mathrm{~cm}^{2}$, thereby exposing $\sim 9 \times 10^{-10}$ mole of $\mathrm{NH}_{2}$ groups at the surface. Yet, the $\sim 6 \mu \mathrm{g} \mathrm{NH}_{3}$ yield corresponds to $3 \times 10^{-7}$ moles of $\mathrm{N}$ atoms.

Thus, the amount of $\mathrm{NH}_{3}$ produced far exceeds the number of $\mathrm{NH}_{2}$ groups exposed on the surfaces. This proves that the formation of $\mathrm{NH}_{3}$ in Fig. 4 results from photocatalytic reduction of $\mathrm{N}_{2}$ dissolved in the aqueous medium, rather than desorption of amino groups or other nitrogen-containing functionalities from the diamond surface. This is further confirmed by 
XPS data shown below.

\subsection{X-ray photoelectron spectroscopy characterization of interfacial stability}
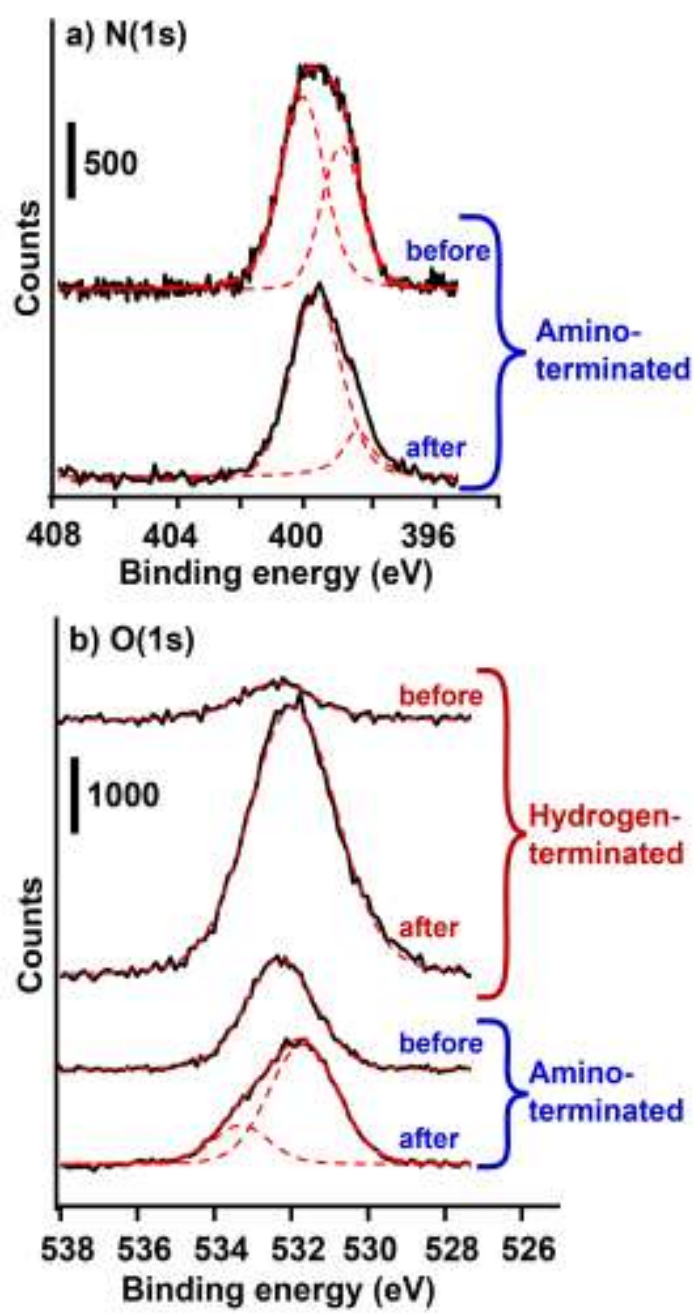

Fig. 5. XPS spectra of amino-terminated and H-terminated diamond before and after 12 hours of illumination with 450 W UV-enhanced HgXe lamp; a) N(1s) spectra from amino-terminated diamond; b) $\mathrm{O}(1 \mathrm{~s})$ from amino-terminated and H-terminated diamond. Data are shown as solid black lines; fits to Voight peaks are shown as dashed red lines. $\mathrm{N}(1 \mathrm{~s})$ spectra were fit to two peaks; $\mathrm{O}(1 \mathrm{~s})$ peaks were fit to one peak except two peaks were necessary to fit the amino-terminated sample after illumination.
In our previous work, we showed that when $\mathrm{H}$-diamond samples are illuminated with ultraviolet light in water, the surface becomes partially oxidized over a time period of several hours. This oxidation leads to positive electron affinity and inhibits electron emission [14]. One motivation for replacing surface $\mathrm{H}$-termination with amino-termination is to increase its durability against free radicals that can be created from solvated electrons in water. In particular, neutral hydroxyl radicals and singlet oxygen atoms are both produced by solvated electrons in water and are known to be able to abstract $\mathrm{H}$ atoms from $\mathrm{C}-\mathrm{H}$ bonds in typical organic molecules $[12,13]$, suggesting that they might also induce loss of H-termination from diamond. We used XPS to investigate the coverage of $\mathrm{N}$ and $\mathrm{O}$ before and after extended $\mathrm{UV}$ irradiation. Fig. 5 shows XPS data for

$\mathrm{H}$-terminated and amino-terminated diamond before and after continuous illumination by a $450 \mathrm{~W} \mathrm{HgXe} \mathrm{lamp} \mathrm{for} 12$ hours in nitrogen-purged water, along with curve fits. The $\mathrm{N}(1 \mathrm{~s})$ spectra require two components to fit, while the $\mathrm{O}(1 \mathrm{~s})$ spectra are precisely fit by a single peak except for the amino-terminated sample after illumination, which shows evidence for 
two $\mathrm{O}(1 \mathrm{~s})$ species. The starting surface shows $\mathrm{NH}_{2}$ groups at approximately $399 \mathrm{eV}$ binding energy, close to the value reported by Wei, et al. for primary amino groups on diamond [21]. Absolute coverages were determined using the $\mathrm{C}(1 \mathrm{~s})$ spectra from the underlying diamond (not shown) as an internal standard as described above show that the surface density of $\mathrm{N}$ atoms remains the same within experimental error $\left(17.3 \pm 2.0\right.$ atoms $/ \mathrm{nm}^{2}$ before $\mathrm{HCl}$ treatment, and $17.1 \pm 2.0$ atoms $/ \mathrm{nm}^{2}$ after) and shows a small shoulder at high binding energies. More notable is the comparison of $\mathrm{H}$-terminated and amino-terminated diamond shown in Fig. 5b; the oxygen coverage on the amino-terminated surface remains essentially unchanged, yielding densities of $20 \pm 2 \mathrm{O}$ atoms $/ \mathrm{nm}^{2}$ before and $17 \pm 2.0$ atoms $/ \mathrm{nm}^{2}$ afterward, while that on the H-terminated surface increased ten-fold, from $(1.9 \pm 0.5$ to $16.7 \pm 2$ oxygen atoms $/ \mathrm{nm}^{2}$ ) in the same amount of time. These data suggest that the amino-terminated diamond shows greater chemical stability under extended UV irradiation in water.

\section{Discussion}

Our results show that amino-terminated diamond surfaces can act as good electron emitters in both vacuum and water, particularly when the amino groups are protonated. Also important is that our data indicate that termination of the surface with amino groups may provide enhanced stability against surface oxidation and resulting loss of NEA.

While amino termination has been investigated previously, earlier studies using plasmas to functionalize diamond surfaces have typically used much more intense plasmas, on the order of 300-500 watts $[42,43]$. However, high plasma powers can lead to extensive dissociation of the ammonia molecules, leading to the formation of cyano groups, amino groups, and other forms of surface nitrogen that are not easy to protonate to form positively charged surface groups. [21,44]. Wei and co-workers reported good results using a microwave plasma, optimized at powers much lower than those typically used for diamond growth [21]. By using a low-power plasma, we minimize fragmentation and achieve a surface terminated with surface densities of amino groups that, when protonated, are sufficient to control the diamond space-charge region $[31,45] /$

While photoemission and ammonia-yield experiments both show that good electron emission 
is observed from the as-prepared $\mathrm{NH}_{2}$-terminated surface, both experiments indicate that electron emission is significantly enhanced by forcibly protonating the surface $-\mathrm{NH}_{2}$ groups to $-\mathrm{NH}_{3}{ }^{+}$groups via exposure to concentrated $\mathrm{HCl}$. The need for rather harsh protonation conditions can be understood on the basis of prior experiments on self-assembled monolayers on silicon and gold surfaces. The propensity for an amino group to be protonated or deprotonated is typically characterized in free solution in terms of its dissociation reaction $\left(\mathrm{R}_{-} \mathrm{NH}_{3}{ }^{+} \rightarrow \mathrm{R}-\mathrm{NH}_{2}+\mathrm{H}^{+}\right)$where the dissociation constant $\mathrm{K}_{\mathrm{a}} \equiv \frac{\left[\mathrm{R}-\mathrm{NH}_{2}\right]\left[\mathrm{H}^{+}\right]}{\left[\mathrm{R}-\mathrm{NH}_{3}^{+}\right]}$and $\mathrm{pK}_{\mathrm{a}} \equiv-\log _{10} \mathrm{~K}_{\mathrm{a}}$. Amino groups within organic and inorganic compounds have $\mathrm{pK}$ values in the range of 9-10 [43] indicating that at $\mathrm{pH}<9$ (or equivalently, $\left[\mathrm{H}^{+}\right]>10^{-9} \mathrm{M}$ ) the amino groups exist primarily in the protonated form $-\mathrm{NH}_{3}{ }^{+}$. However, when charged groups are arranged in a planar array at a surface, Coulombic interactions strongly favor the electrically neutral state, thereby demanding much more acidic conditions to ensure protonation of the amino groups $[42,46-48]$. Whitesides and co-workers found that self-assembled monolayers terminated with $-\mathrm{NH}_{2}$ groups were not fully converted to the protonated form $\left(-\mathrm{NH}_{3}{ }^{+}\right)$even at $\mathrm{pH}<2$ and concluded that this effect arose because the $-\mathrm{NH}_{2}$ groups do not significantly change their hydrophilicity between protonated and deprotonated forms [47]. Others have reported that on the order of $20 \%$ of surface $-\mathrm{NH}_{2}$ groups are protonated near $\mathrm{pH}=7$, but that $\mathrm{pH}<1$ were required to achieve $>50 \%$ protonation of terminal $\mathrm{NH}_{2}$ groups of self-assembled monolayers [43]. While the SDTB method shows that the concentration of primary amine groups is $>6 \times 10^{13} \mathrm{~cm}^{-2}$, this is a lower limit imposed by steric effects in the analysis and also does not include other amine-based functional groups such as secondary amino groups that can also be protonated. Our XPS data show that the surface $\mathrm{Cl}$ concentration is 6.2 atoms $/ \mathrm{nm}^{2}$. If we assume that all $\mathrm{Cl}$ atoms observed in XPS are counter-ions due to protonation of surface amino groups (thus assuring overall charge neutrality), this suggests that there are $\sim 6 \times 10^{14}$ positive charges per $\mathrm{nm}^{2}$ after treatment with $\mathrm{HCl}$. Previous studies of $\mathrm{H}$-terminated diamond in oxygen-containing water have shown that when diamond is immersed in water, the surface Fermi level equilibrates with the $\mathrm{O}_{2} / \mathrm{OH}^{-}$redox couple, leading to upward band-bending and a sub-surface charge density of $\sim 10^{13}$ charges $/ \mathrm{cm}^{2}[31,49]$. Our experiments are conducted under $\mathrm{N}_{2}$-purged conditions and therefore will not equilibrate in the same manner. 
More importantly, the positive charge densities of $\sim 10^{14}$ charges $/ \mathrm{cm}^{2}$ formed from the protonated amino groups far exceeds the charge density reported in those previous studies. Consequently, we anticipate that under the conditions of our experiments the static surface charge associated with the protonated amino groups dominates the electrostatics at the diamond-electrolyte interface. This is further supported by our Mott-Schottky data, which show that amino-terminated surfaces have a substantially more positive flat-band potential compared with H-terminated diamond. This more positive flat-band potential confirms that the amino-terminated surfaces have a more pronounced downward band-bending, as expected for a surface with a net positive charge density.

The XPS data in Fig. 5 show that freshly H-terminated samples show very little oxygen, while samples terminated with amino groups exhibit higher initial $\mathrm{O}(1 \mathrm{~s})$ intensities. We believe the increased $\mathrm{O}(1 \mathrm{~s})$ intensity on amino-terminated surfaces is a consequence of two factors: (1) increased hydrophilicity of amino-terminated surfaces and (2) charge compensation of protonated amino groups $\left(-\mathrm{NH}_{3}{ }^{+}\right)$by hydroxyl groups $\left(\mathrm{OH}^{-}\right)$. Previous studies [50,51] and our own data show that the H-terminated diamond samples are very hydrophobic, while amino-terminated surfaces are very hydrophilic [21]. The more hydrophilic character of amino-terminated surfaces would be expected to lead to increased adsorption of water and higher $\mathrm{O}(1 \mathrm{~s})$ signals, as recently reported by Wei and co-workers [21]. In addition, protonation of $-\mathrm{NH}_{2}$ groups must be accompanied by charge-compensating anions such as $\mathrm{OH}^{-}$or (on $\mathrm{HCl}$-treated samples) by $\mathrm{Cl}^{-}$. The $\mathrm{O}(1 \mathrm{~s})$ intensity on amino-terminated surfaces could also contain contributions from surface-bound $\mathrm{OH}^{-}$groups in the form of $-\mathrm{NH}_{3}{ }^{+} \mathrm{OH}^{-}$ contact-ion pairs. Similar observations and conclusions have been reported in studies of amino-terminated self-assembled monolayers on surfaces of gold [52].

One of the most significant aspects of our work is that amino-termination of the sample appears to significantly enhance the stability of the surface under photochemical reaction conditions. Illumination of the amino-terminated sample in water retains most of the $\mathrm{N}(1 \mathrm{~s})$ peak area, although it is accompanied by subtle changes in the shape of the $\mathrm{N}(1 \mathrm{~s})$ and $\mathrm{O}(1 \mathrm{~s})$ peaks. In the case of the $\mathrm{N}(1 \mathrm{~s})$ peak, it is uncertain if the changes are statistically significant, as the $\sim 1.3 \mathrm{eV}$ difference in binding energy of the two fit components ( 398.3 and $399.6 \mathrm{eV}$ ) is 
less than the average full-width-at-half-maximum of the fit components.

The enhanced stability of amino-terminated diamond compared with $\mathrm{H}$-terminated diamond is likely associated with differences in reactivity of these two surfaces with radicals produced near the electrode surfaces. $\mathrm{O}_{2}$ is an excellent electron scavenger of electrons [13], such that even trace amounts of $\mathrm{O}_{2}$ would be expected to lead to production of reactive species such as the superoxide ion $\left(\mathrm{O}_{2}{ }^{-}\right)$, hydrogen peroxide $\left(\mathrm{H}_{2} \mathrm{O}_{2}\right)$, and neutral hydroxyl radicals $(\mathrm{OH})$ [53]. Hydroxyl radicals are particularly potent oxidizing agents and are known to be able to abstract $\mathrm{H}$ atoms from organic alkanes (lipids) [12,54]. Consequently, we would reasonably expect that hydroxyl radicals would likely be able to abstract $\mathrm{H}$ atoms from $\mathrm{H}$-terminated diamond surfaces, leaving behind reactive C "dangling bonds" that would likely react immediately with water to oxidize the surface. In contrast, abstraction of a proton from a protonated amino group $\left(\mathrm{C}-\mathrm{NH}_{3}{ }^{+}\right)$leaves behind a neutral amino group $\left(\mathrm{C}-\mathrm{NH}_{2}\right)$ that can simply be re-protonated by $\mathrm{H}^{+}$present in ambient water.

\section{Conclusion}

While the vast majority of prior studies of electron emission from diamond have focused on the H-terminated surface, our results show that the amino-terminated surface may provide superior electron emission properties, especially in aqueous environments. Protonation of the surface via exposure to acidic conditions clearly enhances the electron emission even more. The electron emission of diamond from $\mathrm{H}$-terminated diamond is generally attributed to a surface dipole that favors electron emission. In contrast, when using amino-terminated surfaces the electron emission at the surface is enhanced by a sheet of static positive charge just outside of the diamond surface. Since electrostatic interactions with fixed charges are generally stronger than interactions with dipoles, we assert that the use of amino groups may provide an effective way of enhancing electron emission that is also more chemically stable than H-termination.

\section{Acknowledgment.}

This work was supported by the National Science Foundation Grant DMR 1207281 


\section{References}

[1] G.M. Swain, R. Ramesham, Anal. Chem. 65 (1993) 345.

[2] M.C. Granger, et al., Anal. Chem. 72 (2000) 3793.

[3] F.J. Himpsel, J.A. Knapp, J.A. Vanvechten, D.E. Eastman, Phys. Rev. B 20 (1979) 624.

[4] D. Takeuchi, New Diamond Front. Carbon Technol. 15 (2005) 297.

[5] D. Takeuchi, H. Kato, G.S. Ri, T. Yamada, P.R. Vinod, D. Hwang, C.E. Nebel, H. Okushi, S. Yamasaki, Appl. Phys. Lett. 86 (2005) 152103.

[6] J. Vanderweide, Z. Zhang, P.K. Baumann, M.G. Wensell, J. Bernholc, R.J. Nemanich, Phys. Rev. B 50 (1994) 5803.

[7] C. Bandis, B.B. Pate, Phys. Rev. Lett. 74 (1995) 777.

[8] M.J. Rutter, J. Robertson, Phys. Rev. B 57 (1998) 9241.

[9] J.B. Cui, J. Ristein, L. Ley, Phys. Rev. Lett. 81 (1998) 429.

[10] B. Abel, Annu. Rev. Phys. Chem., 64 (2013) 533.

[11] E.J. Hart, S. Gordon, E.M. Fielden, J. Phys. Chem. 70 (1966) 150.

[12] W.R. Haag, C.C.D. Yao, Environ. Sci. Technol. 26 (1992) 1005.

[13] G.V. Buxton, C.L. Greenstock, W.P. Helman, A.B. Ross, J. Phys. Chem. Ref. Data 17 (1988) 513.

[14] D. Zhu, L. Zhang, R.E. Ruther, R.J. Hamers, Nat. Mater. 12 (2013) 836.

[15] R.J. Hamers, J.A. Bandy, D. Zhu, L. Zhang, Faraday Discuss. 172 (2014) 397.

[16] J.R. Christianson, D. Zhu, R.J. Hamers, J.R. Schmidt, J. Phys. Chem. B 118 (2014) 195.

[17] L. Zhang, D. Zhu, G.M. Nathanson, R.J. Hamers, Angew. Chem. Int. Ed. 53 (2014) 9746.

[18] B.D. Thoms, M.S. Owens, J.E. Butler, C. Spiro, Appl. Phys. Lett. 65 (1994) 2957.

[19] A. Kraft, Int. J. Electrochem. Sci. 2 (2007) 355.

[20] C. Chen, B. Liang, D. Lu, A. Ogino, X. Wang, M. Nagatsu, Carbon 48 (2010) 939.

[21] J.J. Wei, J.L. Liu, L.X. Chen, L.F. Hei, F.X. Lv, C.M. Li, Diamond Relat. Mater. 54 (2015) 34 .

[22] S.J. Kang, V.M. Donnelly, Plasma Sources Sci. Technol. 16 (2007) 265. 
[23] R.K. Gaur, K.C. Guptal, Anal. Biochem. 180 (1989) 253.

[24] T. Knickerbocker, T. Strother, M.P. Schwartz, J.N. Russell, J. Butler, L.M. Smith, R.J. Hamers, Langmuir 19 (2003) 1938.

[25] R.A. Franking, E.C. Landis, R.J. Hamers, Langmuir 25 (2009) 10676.

[26] S. Tanuma, C.J. Powell, D.R. Penn, Surf. Interface Anal. 37 (2005) 1.

[27] J.F. Moulder, W.F. Stickle, P. Sobol, K.D. Bomben, Handbook of X-ray Photoelectron Spectroscopy, Physical Electronics, Inc., 1995.

[28] L. Diederich, O. Kuttel, P. Aebi, L. Schlapbach, Surf. Sci. 418 (1998) 219.

[29] B.A. Putans, L.M. Bishop, R.J. Hamers, Chem. Mater. 26 (2014) 3651.

[30] V. Privman, J.S. Wang, P. Nielaba, Phys. Rev. B 43 (1991) 3366.

[31] C.E. Nebel, B. Rezek, A. Zrenner, Diamond Relat. Mater. 13 (2004) 2031.

[32] S. Busch, L.C. Pardo, W.B. O'Dell, C.D. Bruce, C.D. Lorenz, S.E. McLain, Phys. Chem. Chem. Phys. 15 (2013) 21023.

[33] P.K. Baumann, R.J. Nemanich, J. Appl. Phys. 83 (1998) 2072.

[34] L. Kronik, Y. Shapira, Surf. Sci. Rep. 37 (1999) 1.

[35] P. Allongue, H. Cachet, J. Electrochem. Soc. 132 (1985) 45.

[36] H. Gerischer, J. Electroanal. Chem. 150 (1983) 553.

[37] J.E.B. Randles, Discuss. Faraday Soc. 1 (1947) 11.

[38] N.G. Ferreira, L.L.G. Silva, E.J. Corat, V.J. Trava-Airoldi, K. Iha, Brazilian J. Phys. 29 (1999) 760.

[39] F. Cardon, W. Gomes, J. Phys. D: Appl. Phys 11 (1978) L63.

[40] R. De Gryse, W.P. Gomes, F. Cardon, J. Vennik, J. Electrochem. Soc. 122 (1975) 711.

[41] K.-Y. Tse, B.M. Nichols, W. Yang, J.E. Butler, J. John N. Russell, R.J. Hamers, J. Phys. Chem. B 109 (2005) 8523.

[42] K.P. Fears, S.E. Creager, R.A. Latour, Langmuir 24 (2008) 837.

[43] F. Milletti, L. Storchi, L. Goracci, S. Bendels, B. Wagner, M. Kansy, G. Cruciani, Eur. J. Med. Chem. 45 (2010) 4270.

[44] A. Denisenko, A. Romanyuk, L.A. Kibler, E. Kohn, J. Electroanal. Chem. 657 (2011) 164.

[45] M.T. Edmonds, C.I. Pakes, S. Mammadov, W. Zhang, A. Tadich, J. Ristein, L. Ley, Appl. 
Phys. Lett. 98 (2011).

[46] H. Munakata, D. Oyamatsu, S. Kuwabata, Langmuir 20 (2004) 10123.

[47] S.R. Holmes-Farley, C.D. Bain, G.M. Whitesides, Langmuir 4 (1988) 921.

[48] H. Zhang, H.X. He, J. Wang, T. Mu, Z.F. Liu, Appl. Phys. A 66 (1998) S269.

[49] J.A. Garrido, A. Hartl, M. Dankerl, A. Reitinger, M. Eickhoff, A. Helwig, G. Muller, M. Stutzmann, J. Am. Chem. Soc. 130 (2008) 4177.

[50] L. Ostrovskaya, V. Perevertailo, V. Ralchenko, A. Dementjev, O. Loginova, Diamond Relat. Mater. 11 (2002) 845.

[51] I. Yagi, H. Notsu, T. Kondo, D.A. Tryk, A. Fujishima, J. Electroanal. Chem. 473 (1999) 173.

[52] J.E. Baio, T. Weidner, J. Brison, D.J. Graham, L.J. Gamble, D.G. Castner, J. Electron Spectrosc. Relat. Phenom. 172 (2009) 2.

[53] T.L. Thompson, J.T. Yates, Chem. Rev. 106 (2006) 4428.

[54] K. Anzai, K. Ogawa, Y. Goto, Y. Senzaki, T. Ozawa, H. Yamamoto, Antioxid. Redox Signaling 1 (1999) 339. 


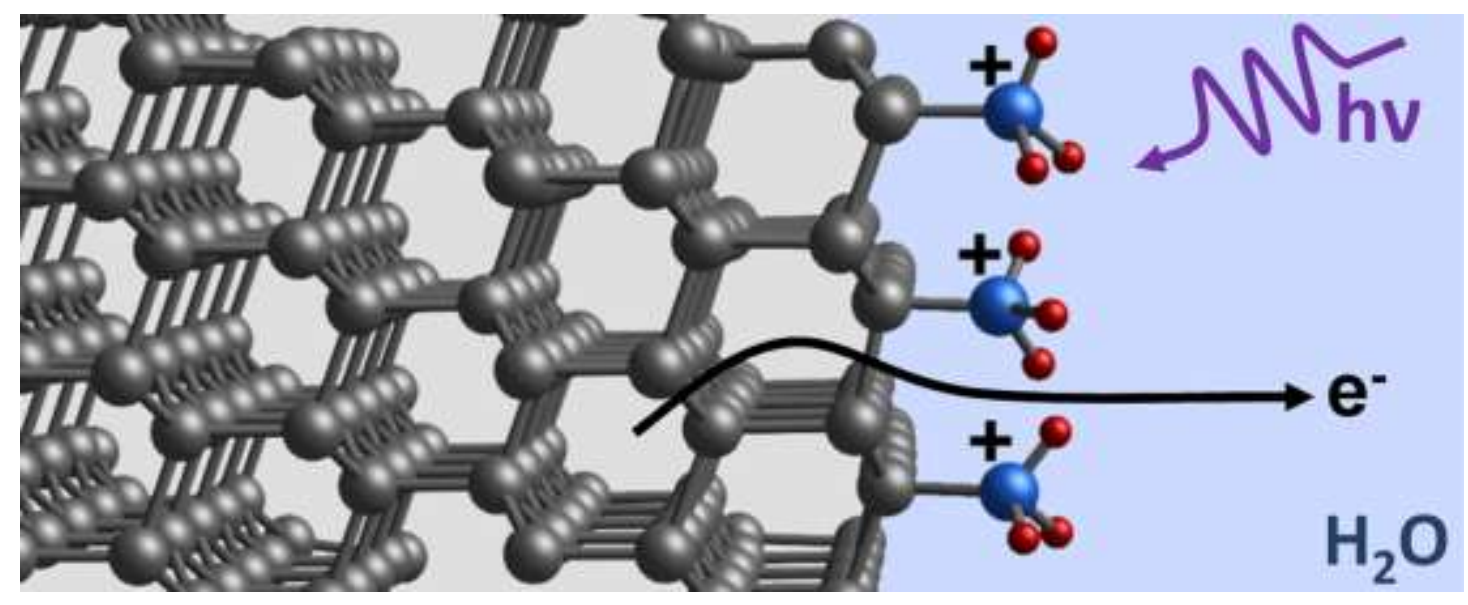

